\title{
Large-eddy simulation of unsteady turbine rim sealing flows
}

\author{
Feng Gao ${ }^{\mathrm{a}, *}$, John W Chew ${ }^{\mathrm{a}}$, Paul F Beard ${ }^{\mathrm{b}}$, Dario Amirante ${ }^{\mathrm{a}}$, Nicholas J Hills ${ }^{\mathrm{a}}$ \\ ${ }^{a}$ Faculty of Engineering and Physical Sciences, University of Surrey, Guildford GU2 7XH, UK \\ ${ }^{b}$ Osney Thermo-Fluids Lab., Dept. of Eng. Sci., University of Oxford, Oxford, OX2 OES. UK
}

\begin{abstract}
Unsteady flow phenomena unrelated to the main gas-path blading have been identified in a number of turbine rim seal investigations. This unsteadiness has significant influence on the sealing effectiveness predicted by the conventional steady RANS (Reynolds-averaged Navier-Stokes) method, thus it is important for turbine stage design and optimisation. This paper presents CFD (computational fluid dynamics) modelling of a chute type rim seal that has been previously experimentally investigated. The study focuses on inherent large-scale unsteadiness rather than that imposed by vanes and blades or external flow. A large-eddy simulation (LES) solver is validated for a pipe flow test case and then applied to the chute rim seal rotor/stator cavity. LES, RANS and unsteady RANS (URANS) models all showed reasonable agreement with steady measurements within the disc cavity, but only the LES shows unsteadiness at a similar distinct peak frequency to that found in the experiment, at 23 times the rotational frequency. The boundary layer profile within the chute rim seal clearance has been scrutinised, which may explain the improvement of LES over RANS predictions for the pressure drop across the seal. LES results show a clockwise mean flow vortex. A more detailed sketch of the rim sealing flow unsteady flow structures is established with the help of the LES results. However, there are some significant differences between unsteadiness predicted and the measurements, and possible causes of these are discussed.
\end{abstract}

Keywords: turbine rim seal, Chute seal, unsteady flow structures, large-eddy simulation, Taylor-Couette instability

PACS: 47.20.Qr, 47.27.ep

\section{Introduction}

Turbine rim sealing flows are a key issue in turbomachinery design, affecting both turbine aerodynamic losses and turbine rotor disc lifetime. A typical rim seal arrangement includes a narrow azimuthal clearance between rotor and stator disc platform, connecting the rotor/stator disc cavity and the main gas path. Additional cooling air is required to prevent ingestion of the hot main annulus gas into the turbine disc cavity which, if allowed, may lead to thermal failure of the rotor disc. In addition, pumping too much cooling air into the main gas path may result in aerodynamic losses. Therefore, correct modelling of sealing effectiveness is desired for turbomachinery design and its optimisation. However, modelling of such flows with Reynolds-averaged Navier-Stokes (RANS) has proved difficult and several experimental and numerical studies have indicated that the rim seal gap and disc cavity flows can contain large scale unsteady flow structures with frequencies unrelated to those associated with the rotating blades.

Basic mechanisms involved in rim sealing flow phenomena were classified by Johnson et al. [1]. Among these are (1) disc pumping effect, (2) three-dimensional (3-D) and time dependent periodic pressure field created by vanes and blades, (3) 3-D geometry within rim seal region, (4) asymmetries in the rim seal geometry, (5) turbulent transport in the platform overlapping region, and (6) flow entrainment.

\footnotetext{
* Corresponding author

Email addresses: f.gao@surrey.ac.uk (Feng Gao), j.chew@surrey.ac.uk (John W Chew), paul.beard@eng.ox.ac.uk (Paul F Beard), d.amirante@surrey.ac.uk (Dario Amirante), n.hills@surrey.ac.uk (Nicholas J Hills)
} 
In addition to these basic mechanisms mentioned above, a number of experimental and numerical studies have reported large-scale low-frequency unsteady flow structures in the rim seal gap and disc cavity which are not attributed to the main gas-path vanes and blades. The first evidence of this kind of unsteadiness emerged in the early 2000s. In 2002, Smout et al. [2] mentioned the possible presence of large-scale lowfrequency pressure fluctuations having larger wave length than that associated with the pitch of the vanes or blades, in the Aachen University 1.5 stage turbine rim sealing test rig designed by Bohn et al. [3]. The first published experimental evidence of the large-scale unsteady flow features was reported by Cao et al. [4] in 2003, who also showed some agreement with unsteady RANS (URANS) solutions. Prior to this, in 2002, Autef [5] reported URANS solutions showing unsteady flow structures of a rim seal configuration without vanes and blades, as described by Chew et al. [6]. In 2004 Jakoby et al. [7] reported URANS studies showing large-scale unsteady flow structures unrelated to the blade passing, and supported by measurements from Bohn et al.'s rig.

The above studies relate to axial clearance seals. Boudet et al. [8] reported URANS solutions for a chute seal geometry. These revealed that the flows in this configuration were also inherently 3-D and unsteady. The authors attributed this phenomenon to the possible Taylor-Couette instability in the seal. O'Mahoney et al. [9] extended the turbine stage URANS study of Boudet et al. to LES, and showed closer agreement of sealing effectiveness with the experiment of Gentilhomme [10].

Other researchers have also confirmed the presence of non-blade passing related unsteadiness associated with rim seals, using both computational fluid dynamics (CFD) and through experiment. Schuepbach et al. [11] claimed that the asymmetric pressure field induced by the large-scale flow features can significantly reduce engine performance. Chilla et al. [12] reported strong unsteady flow interaction between the rim seal and the main gas path at nominal sealing flow conditions, and periodically vortex shedding from rim seal into the main annulus. Rabs et al. [13] identified similar vortex structures and conjectured that they could be induced by the Kelvin-Helmholtz instabilities. The latest investigations have all experimentally confirmed the existence of rim seal cavity modes which are unattributed to the blade passing. Amongst recent experimental studies are papers by Beard et al. [14], Savov et al. [15], and Schädler et al. [16]. The study by Beard et al. revealed, for the first time, the speed and number of flow structures independently of CFD solutions.

A number of researchers have developed "orifice models" for rim sealing flows. These models estimate the inflow and the outflow through the seal, taking account of the pressure assymetry in the main annulus. A recent example of this type of model is that developed at the University of Bath [17]. With appropriate choice of model parameters it shows a good scaling capability in correlating measured sealing effectiveness for various seal configurations. Hills et al. [18] developed an orifice model to consider the pressure asymmetries due to both blades and vanes, with inclusion of a term to account for inertial effects. This showed the significance of unsteady flow effects but does not represent the effects of the low frequency unsteadiness discussed above. The unsteady RANS model of Boudet et al. [8], which shows inherent unsteady flow features of the rim seal flow, achieves significant improvement in agreement with measured sealing effectiveness to the steady RANS. Thus, it can be conjectured that correct modelling of unsteady rim sealing flow structures is essential for the accurate prediction of sealing effectiveness, and that current design methods do not capture some important flow physics.

From recent publications it is clear that the detailed flow physics in rim seals is of considerable interest, with a need for better understanding of the underlying flow mechanisms. The present study focuses on the inherent unsteadiness involved in the rim seal, and considers CFD modelling of the chute rim seal geometry published in [14]. Wall resolved URANS and LES solutions are presented and discussed.

\section{Experimental and numerical configurations}

\subsection{Experimental rig}

A sectional view of the Oxford rotor facility (ORF) used by Beard et al. [14] is illustrated in Fig. 1 (a) and (b). In this build, turbine vanes and blades are replaced by platform rings, in order to focus on the inherent flow physics involved in the rim seal configuration. The design of the rim seal followed the chute 
seal of Gentilhomme [10]. The nominal seal gap is $1 \mathrm{~mm}$ with $2 \mathrm{~mm}$ axial overlap, and the rim seal angle is $20^{\circ}$ to the shaft axis.

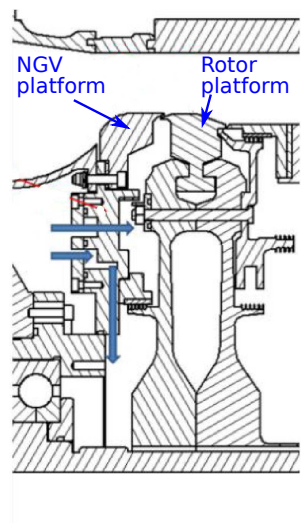

(a)

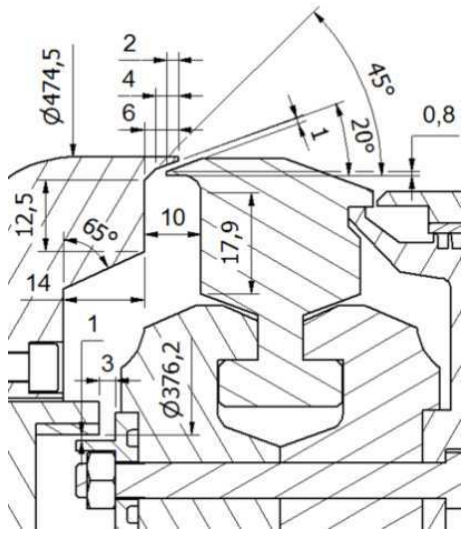

(b)

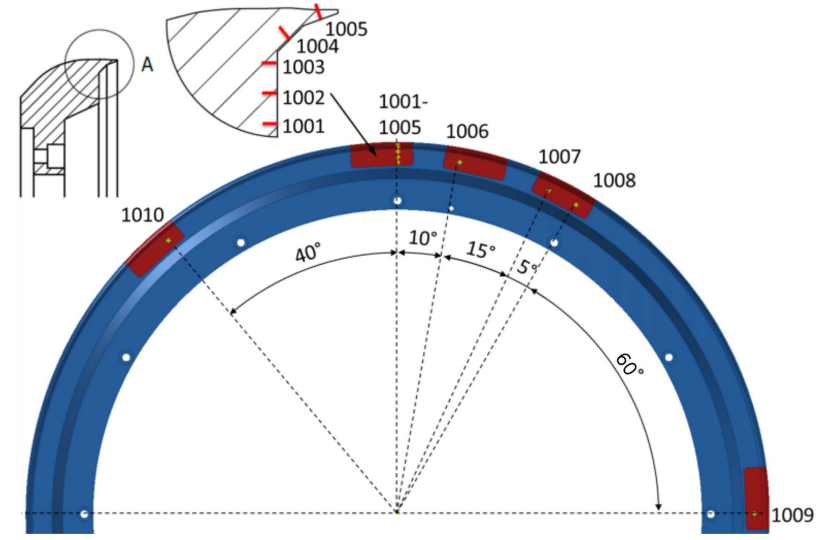

(c)

Figure 1: (a) Schematic of the ORF working section, (b) Rim seal geometry (dimensions in mm), (c) unsteady pressure sensor instrumentation on the vane platform ring (viewed from downstream).

Pressure sensors were embedded in the vane platform ring to record the steady and unsteady static pressure, and the illustration is shown in Fig. 1 (c). The radial and circumferential locations of the fastresponse sensors are listed in Tab. 1. Azimuthal angles are defined in a clockwise direction from top-deadcenter viewing from the rotor. For the steady pressure measurement, five pressure tappings were radially installed on the azimuthal location of $180^{\circ}$ at the same radii as sensors 1001 to 1005 in Tab. 1. For the unsteady pressure measurement, 10 Kulites have been employed to record unsteady pressure signals. They were installed at the positions 1001-1005 and at additional five circumferential locations having the same radius as the sensor 1002. Further details about the rig, pressure measurement uncertainties and the test matrix are given by Beard et al. [14]. As reported by Beard et al., similar unsteady flow features were identified over a range of rotor speeds and sealing flow rates.

Table 1: Pressure sensor locations

\begin{tabular}{lll||lll}
\multicolumn{5}{c}{ Table 1: Pressure sensor locations. } \\
\hline Sensor & Radius $(\mathrm{mm})$ & Angle $\left(^{\circ}\right)$ & Sensor & Radius $(\mathrm{mm})$ & Angle $\left(^{\circ}\right)$ \\
\hline 1001 & 224 & 0 & 1006 & 227.5 & 10 \\
1002 & 227.5 & 0 & 1007 & 227.5 & 25 \\
1003 & 231 & 0 & 1008 & 227.5 & 30 \\
1004 & 234.1 & 0 & 1009 & 227.5 & 90 \\
1005 & 235.9 & 0 & 1010 & 227.5 & -40 \\
\hline
\end{tabular}

\subsection{CFD modelling and numerical settings}

Wall resolved URANS and LES models are reported for the geometry described above at a rotor speed of 7000rpm and without any imposed seal flow, corresponding to the experimental condition at $R e_{\phi}=2.2 \times 10^{6}$ and $C_{w}=0$. Simulations were carried out using a modified version of the Rolls-Royce plc in-house CFD solver Hydra. This code is an unstructured, node-based finite volume solver for compressible Navier-Stokes equations in Cartesian coordinates, and is parallelised using the OPLUS library (Oxford parallel library for unstructured solver) [19]. The URANS and LES models are discussed below. 


\subsubsection{URANS}

The URANS mesh was initially generated in a 2D plane (as shown in Fig. 2) and extruded into a $30^{\circ}$ sector $^{1}$, using ICEMCFD. The computational sector size is larger than the space to accommodate one lobed flow structure identified in the experiment, and is representative of what might be used in a design process where the lobe number is unknown. The near wall grid size is set to $\Delta y^{+} \approx 1$ with circumferential grid spacing being $0.5^{\circ}$ (61 nodes), yielding a total of $\sim 1.6$ million mesh nodes. In the experiment, the main annulus was open to the laboratory with no forced flow raising difficulties for the CFD boundary conditions, as reverse flows were likely at the inlet and the outlet. Therefore, all the inlets and the outlet in the CFD model are configured as inviscid walls for the zero coolant flow case considered, forming a sealed model. Other boundaries are set as isothermal no-slip walls with a fixed static temperature (288K) and the corresponding rotational speed. Circumferential boundaries are connected by a periodic condition. Fluxes are interpolated using a $2^{\text {nd }}$-order centred scheme with a $2^{\text {nd }}$-order smoothing for the inviscid terms. The RANS equations are closed by the Spalart-Allmaras turbulence model [20]. The URANS simulation is initialised by a steady RANS solution.

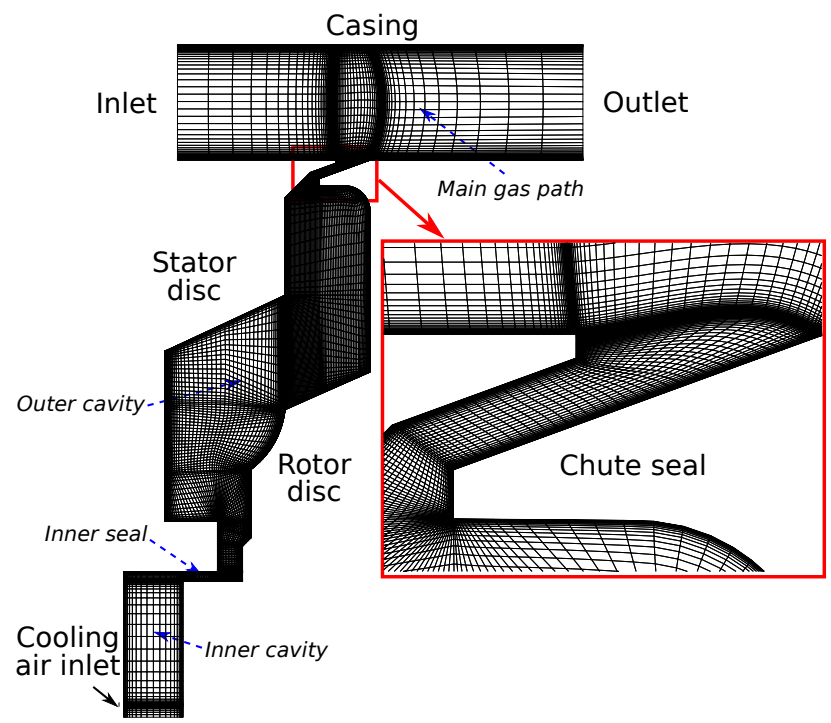

Figure 2: Meridional view of mesh for URANS.

A dual-time stepping implicit temporal scheme is employed for URANS. The physical time step is set to 1/7200 times rotor revolution time, corresponding to a Courant-Friedrichs-Lewy (CFL) number of 2976 in the smallest grid cells. A five-step Runge-Kutta scheme is used for inner iteration convergence acceleration. As suggested by Jameson [21], a physical time step at $\mathrm{CFL}=4000$ could converge to a sufficient accuracy within 10-15 multi-grid cycles with an inner CFL number 5-8. This yields a speed-up ratio of 33-80 compared with an explicit temporal scheme. A smaller speed-up ratio is expected in this study as the multi-grid scheme is not utilised. Therefore, the sub time step number is set to 100, corresponding to a speed-up ratio of about 15 under an inner CFL number of 2.

\subsection{2. $L E S$}

The LES of the chute rim seal flow has the same geometry as for the RANS modelling presented above, but without the inner cavity beneath the inner seal (where no unsteady flow effects were seen in the URANS

\footnotetext{
${ }^{1}$ A slight geometric discrepancy exists between the experiment and CFD geometry. The edge with $45^{\circ}$ slope connected to the chute rim seal boundary on the stator (as shown in Fig. 1 (b)) moved horizontally toward the rotor by $1 \mathrm{~mm}$ in the CFD modelling, due to a confusion with a previous rig design. This is expected to have little effect on the results, as confirmed by the comparison of the steady pressure distribution in Fig. 5.
} 
simulation), in order to reduce the computing costs. The mesh is refined to reach the LES requirement: wall normal distance $\Delta y^{+} \approx 1$ at near wall grids, streamwise grid size $\Delta x^{+}<40$ in the meridional plane, and circumferential grid size $\Delta(r \theta)^{+} \leq 100$ on the rotor rim. In this study, the LES mesh is coarsened in the circumferential direction $\left(\Delta(r \theta)^{+} \leq 160\right)$ to reduce computing costs. As about 27 lobed flow perturbation cells were observed in the experiment, the sector angle is set to $13.33^{\circ}$ to contain at least one lobe in the computational zone. This yields a total of $\sim 6.2$ million mesh nodes.

The Rolls-Royce plc in-house CFD code Hydra was initially developed by Moinier [22], and has recently been extended to LES with $2^{\text {nd }}$-order accuracy by Amirante and Hills [23] using monotone upwind schemes for conservation laws (MUSCL) with linear reconstructions of the primitive variables. The Roe scheme is employed for spatial flux discretisation, and its weighting coefficient of numerical viscosity $\varepsilon$ is set to its minimum value possible $(\varepsilon=0.005)$ to minimise the artificial dissipation and to assure numerical stability. Time integration is performed using an explicit 3-step Runge-Kutta scheme. The standard Smagorinsky subgrid-scale (SGS) model with a van Driest damping function is implemented to predict the subgrid-scale behaviours. Although researchers [24] suggested a Smagorinsky model coefficient of $C_{s}=0.1$, the present authors adopt the value $C_{s}=0.08$ suggested by $\mathrm{Li}$ [25] through an a priori analysis of SGS model based on DNS databases. This solver is validated for a pipe flow test case and then applied to a sectored model of the disc cavity and the chute rim seal.

\section{Validation}

\subsection{Validation of the Hydra LES code}

A DNS (direct numerical simulation) study of a pipe flow by Khoury et al. [26], at $R e_{\tau}=1000$, is considered as reference to validate the LES solver. Non-dimensional parameters provided in the literature and dimensional variables used in this study are listed in Tab. 2.

Table 2: Parameters for pipe flow test case.

\begin{tabular}{ll||ll}
\hline \multicolumn{2}{l||}{ Non-dimensional parameters } & \multicolumn{2}{c}{ Dimensional parameters } \\
\hline Parameter & Value & Parameter & Value \\
\hline$R e_{b}$ & 37700 & Radius $(R)$ & $4.17 \times 10^{-3} \mathrm{~m}$ \\
$R e_{\tau}$ & 1000 & Length & $15 R$ \\
$u_{\tau} / u_{b}$ & 0.053 & Bulk velocity $\left(u_{b}\right)$ & $70 \mathrm{~m} / \mathrm{s}$ \\
- & - & Friction velocity $\left(u_{\tau}\right)$ & $3.71 \mathrm{~m} / \mathrm{s}$ \\
\hline
\end{tabular}

The near wall grid spacing in the radial direction is set to be less than 1 wall unit $\left(\rho u_{\tau} \Delta r / \mu\right)$, and the maximum spacings are about 80 and 40 wall units in the streamwise and circumferential directions. An isothermal no-slip wall condition is applied to the pipe surface, and streamwise boundaries are connected by a periodic condition. A variable body force is applied to the flow field to maintain a constant mass flow rate, following the procedures of Lenormand et al. [27]. The simulation is initialised with a laminar parabolic Poiseuille velocity profile with a random velocity fluctuation up to $30 \%$ of its mean value, as described by Eq. (1). Five flow passing periods through the pipe are used to reach the transition and steady state, and ten periods are simulated to converge the statistics.

$$
v_{z}(r, t=0)=2 u_{b}\left(1-\left(\frac{r}{R}\right)^{2}\right)(1+0.3 \xi)
$$

where $\xi \in[-1,1]$ is a random number.

The comparison of velocity profile and Reynolds stresses with the DNS data is shown in Fig. 3. Very good agreement of the mean streamwise velocity profile with the DNS results is achieved by the present LES, and the LES Reynolds stresses agree reasonably well with DNS. 


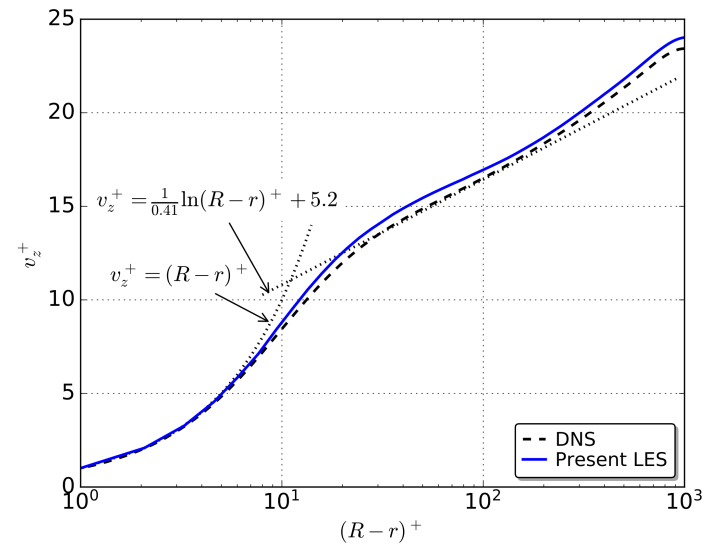

(a)

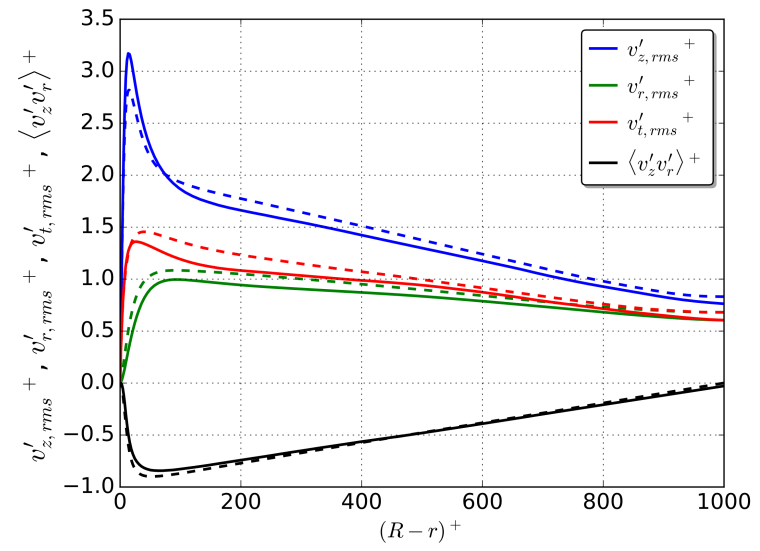

(b)

Figure 3: Mean velocity (a) and Reynolds stress (b) profiles of a pipe flow at $R e_{\tau}=1000$.

\subsection{Validation of rim sealing flow simulation}

The LES of the rim sealing flow simulation is initialised with a converged RANS solution. Five rotor revolution periods are simulated to reach a steady state, and eight rotor rotational periods are used to converge the statistics. The statistical convergence of static pressure on the five radial sensors is plotted in Fig. 4. The mean pressures are well converged, and high order statistics reach a satisfactory convergence.

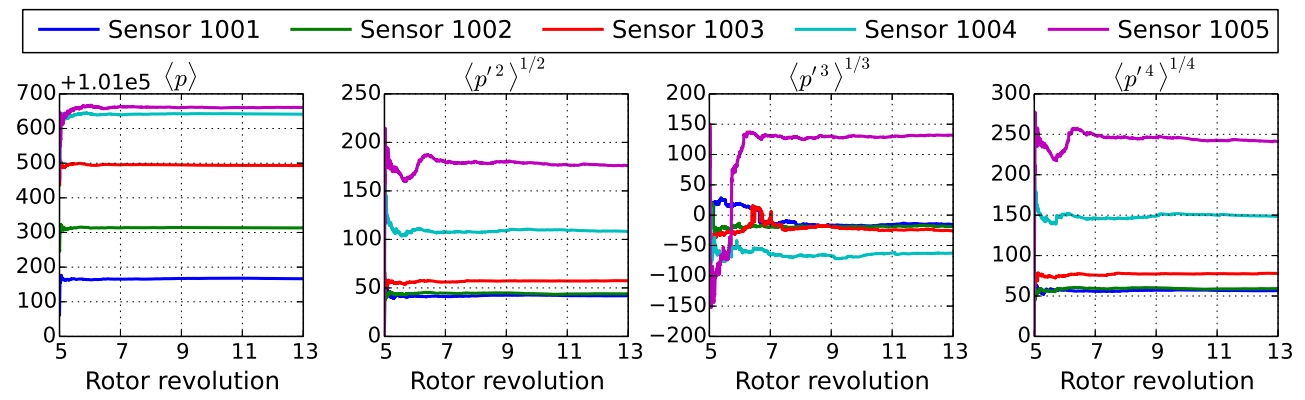

Figure 4: Statistical convergence of static pressure on the five radially distributed sensors.

The validation and convergence check of the solver and LES modelling provide confidence for further analysis and discussion of the results.

\section{Results and discussions}

In this section, the RANS and LES solutions are further validated against the mean and unsteady pressure measurements. Then the mean velocity profiles within the rim seal gap are scrutinised. Finally the unsteady flow characteristics are deduced and discussed in detail.

\subsection{Mean Pressure Distribution}

Whilst the aim of this study is to investigate the unsteady flow structures involved in the rim seal, the mean pressure distribution is also of interest. The mean pressure is presented here as the pressure difference to the sensor 1001 normalised by the dynamic pressure at disc rim speed, as expressed in Eq. (2). In the experiment, the pressure differences were less than $1 \%$ of the atmospheric pressure and the variation of temperature is also relatively small, so fluid density and viscosity are assumed to be constant and their 
values were computed from the atmospheric pressure and the coolant flow temperature which is close to atmospheric conditions. In the simulations, fluid density and viscosity are nearly constant as well. The reference density is taken as the averaged value at the five radial sensor locations.

$$
C_{p}=\frac{\langle p\rangle-\left\langle p_{1}\right\rangle}{0.5 \rho \Omega^{2} b^{2}}
$$

The mean static pressure distributions from the experiment, steady RANS, LES and URANS are plotted in Fig. 5. The radii of the pressure sensors are normalised by the disc rim radius. The main flow path is open to the atmosphere in the experiment, so the experimental outer annulus pressure is approximately uniform. This and the CFD annulus pressures are plotted at $r / b=1.01$. In the sealed CFD system, annulus pressures near the casing and hub are different, therefore both of them are plotted to compare with the experiment. A forced vortex pressure distribution at 55\% rotor speed is also plotted in the figure. In the cavity all of the results follow the forced vortex distribution, indicating that the mean pressure is dominated by the flow rotation. All the CFD results show good agreement with experiment at the three innermost sensor positions in the cavity, while a significant improvement of the prediction near the chute seal has been achieved by LES. The RANS and LES pressure at rotor hub in the annulus are close to the experimental atmospheric pressure. The URANS mean pressure follows the RANS one in the cavity at the four inner sensor position, and appears to give a slight improvement over RANS within the chute seal.

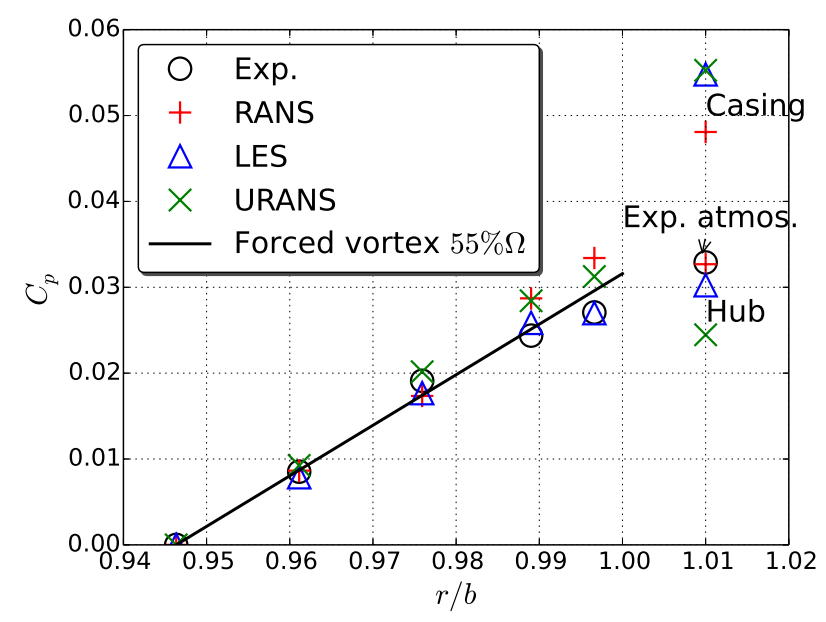

Figure 5: Mean static pressure distribution.

Both the LES and (U)RANS results agree with measurements within the cavity. Considering the pressure difference across the rim seal, LES gives the best agreement with the measurements. This may be associated with the mean velocity profiles and unsteady flow in the rim seal, as will be discussed later.

\subsection{Frequency spectra}

In order to detect large-scale unsteady flow structures, high resolution pressure measurements were recorded from 10 fast response sensors (see Fig. 1 (c)) during 1 second, corresponding to about 116 rotor revolutions. High amplitude spectral frequencies at 30 and 60 times disc speed observed in the experiment that related to the 30 bolts beneath the inner seal have been filtered out for results presented in this paper. LES results are stored over 8 rotor revolutions using probes at radial positions corresponding to the experimental pressure sensors with $5^{\circ}$ circumferential spacing. For URANS, unsteady pressure data have been recoded over 4 rotor revolutions using probes with $20^{\circ}$ spacing at the same radial locations as in the experiment and LES.

The power spectral density (PSD) is employed to calculate the frequency spectra. The unsteady pressure signals are split into 25 windows for the experiment and 2 segments for the LES and URANS (corresponding 
to about 4 rotor revolutions per window for LES, and 2 rotations per segment for URANS), with the segmented signal being zero padded to twice its original length and $50 \%$ overlapping between segments.

The PSD results are plotted in Fig. 6 for the radially distributed pressure sensor positions. Distinct peaks at frequency $f / \Omega=23.4$ are observed in the experimental results for the sensors from 1001 to 1004, while a higher amplitude with a wider frequency range is detected by sensor 1005 within the chute seal clearance. In the LES, only the sensors 1004 and 1005 show distinct peaks. Comparing the LES results with those of the experiment, one should notice that an excellent agreement of distinct peak frequency $(f / \Omega \approx 23.5)$ has been achieved. This suggests that about 23.5 periodic flow structures have been captured during a rotor revolution time, by both the experiment and LES. Some discrepancies are observed as well. Within the seal clearance (sensor 1005) more pronounced unsteadiness with a wide range of frequencies is indicated by the experiment. This broadband unsteadiness is not observed in the LES results. Another two distinct peaks are found at $f / \Omega=36.5$ and 47 in the LES results, but were not observed in the experiment. The PSD of URANS results shows two peaks at $f / \Omega=16.2$ and 21.7 for the two uppermost sensor positions. Thus the LES gives closer agreement with experiment than URANS in terms of the main unsteadiness frequency.

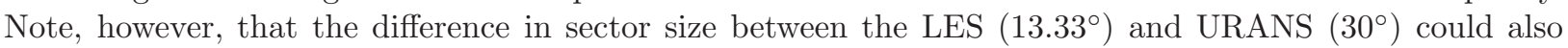
have influenced the frequency.

A number of factors may contribute to differences between the LES and experimental results, and these are to be investigated in further studies. As reported by Beard et al. [14] the unsteady pressure measurements showed effects of boltheads in the inner cavity on the rig (shown in Fig. 1 (b)). The experiments were also subject to a small eccentricity giving deviations in the seal gap of up to $8 \%$. Neither of these effects were included in the LES which also imposed periodicity on a $13.33^{\circ}$ sector. A further consideration is that the outer annulus was open to the laboratory environment, while a sealed annulus was assumed for the LES. Rotating flows are known to be susceptible to waves and the LES results indicate that the chute seal can give rise to such flows. In contrast to the LES, the experiments show the amplitude of the distinct frequency reducing as the chute seal region is approached. However, the measured broadband unsteadiness in the chute region shows more fluctuating energy than observed at lower radii. The broadband energy could, for example, be due to eccentricity while the growth of distinct frequency signal moving away from the seal might be associated with resonance involving interaction with the inner seal, inner disc cavity and/or outer annulus flow. Further research is needed to clarify such effects.

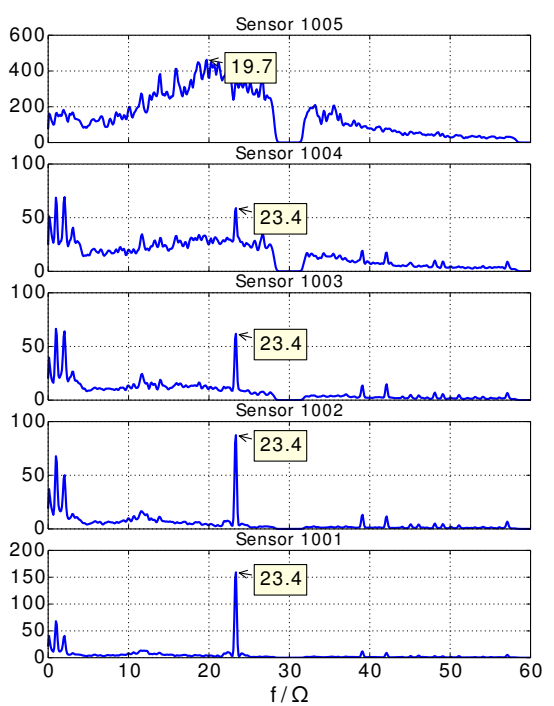

(a)

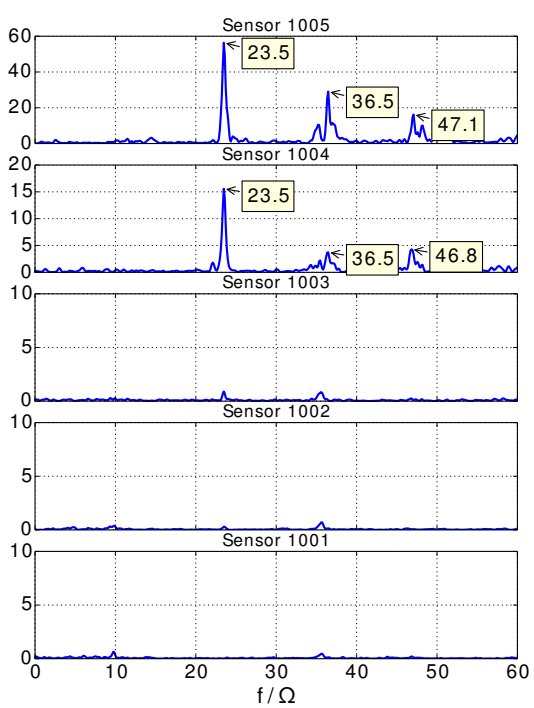

(b)

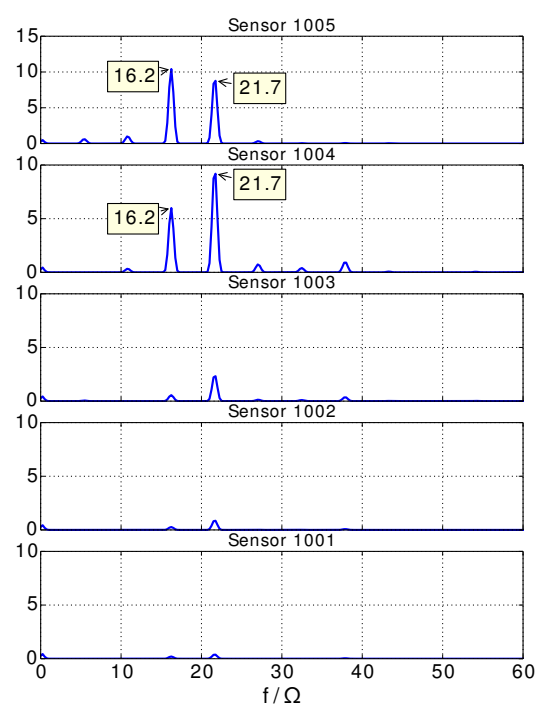

(c)

Figure 6: PSD of pressure data on radially distributed sensors. (a) experiment; (b) LES; (c) RANS. 


\subsection{Mean velocity profiles}

As discussed above, the mean pressure distribution is dominated by the disc rotation which drives the tangential velocity in the core flow between the rotor and stator. More complex flow is expected within the rim seal gap. Considering this region velocity vectors are decomposed into streamwise component $u_{s}$, the wall-normal component $u_{n}$ and tangential component $u_{\theta}$. The averaged tangential components in the seal region and the $s-n$ coordinate system are illustrated in Fig. 7, on a meridian plane. The RANS, URANS and LES results are compared on a number of sampling lines. In Fig. 7 (a), classic rotor-stator swirl velocity profiles are observed, i.e. $0 \mathrm{~m} / \mathrm{s}$ on the stator surface and rising to $\Omega r$ on the rotor surface, with a wide core region with a swirl velocity magnitude around $40 \%$ to $50 \%$ of rotor speed. Slight differences are observed between the three solutions. A zoomed view of the tangential velocity profiles on the three sampling lines labelled from A to C is shown in Fig. 7 (b). It is clear that the RANS results give the highest core swirl velocity, and that of the LES is the weakest. This is consistent with the mean pressure distribution in Fig. 5 where the LES has the smallest $C_{p}$ within the overlapping rim seal region, according to radial equilibrium theory. From the sampling line A to $\mathrm{C}$, the core tangential velocity decreases.

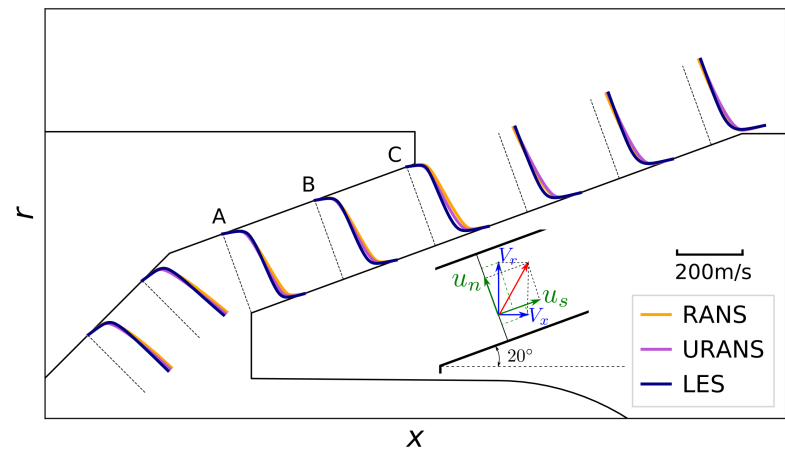

(a)

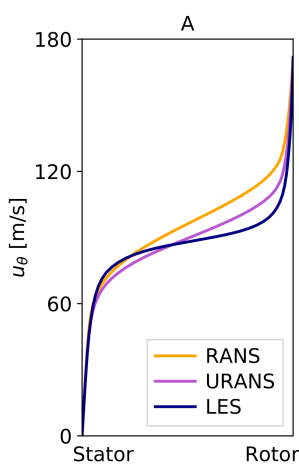

Rotor

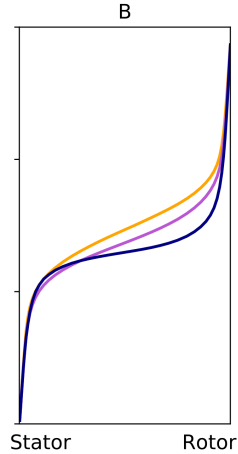

(b)

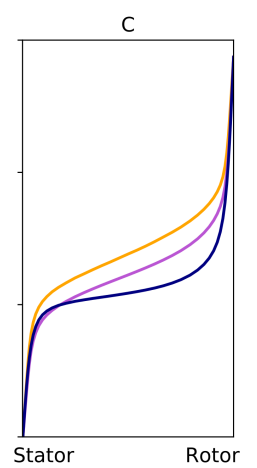

Figure 7: Mean tangential velocity profiles $u_{\theta}$ within the seal gap. (a) view on the geometry; (b) zoom on the three labelled sampling lines.

In a rotor-stator cavity, the fluid near the rotor surface flows outboard from the shaft axis due to the rotor's pumping effect, and flows towards the shaft axis near the stator surface. This forms a classic vortex on the meridian plane, and this behaviour is usually observed through the radial velocity component. To scrutinise this phenomenon in the rim seal clearance, the averaged streamwise velocity $\left(u_{s}\right)$ profiles in parallel to the stator or rotor surface are depicted in Fig. 8. Striking differences are observed between the RANS, URANS and LES results. Outside the overlapping rim seal region, classic rotor-stator velocity profiles are observed, i.e. positive near the rotor surface and negative near the stator surface. Within the overlapping gap, RANS shows the classic behaviour of the rotor-stator cavity, whereas URANS and LES present unusual streamwise velocity profiles, i.e. negative on the rotor and positive on the stator. This implies that the mean ingestion occurs near the rotor, while the mean egestion takes place near the stator. This phenomenon is most pronounced in LES results. On the sampling line $\mathrm{C}$, the mean ingestion and egestion velocity amplitudes reach their maxima. In contrast to the velocity profiles outside this area, URANS and LES show a 2-D clockwise vortex on this meridian plane within the seal gap. This suggests the presence of a Taylor-Couette vortex in the mean flow. A possible Taylor-Couette flow mechanism in the rim sealing flow was also suggested by Boudet et al. [8].

\subsection{Unsteady flow structures}

The mean characteristics of the large scale unsteady flow structures involved in the rim sealing flow are deduced in this section with the help of circumferentially distributed pressure probes in both the experiment and CFD. Then the instantaneous flow fields are analysed in detail to illustrate some features of the unsteady flow structures. 


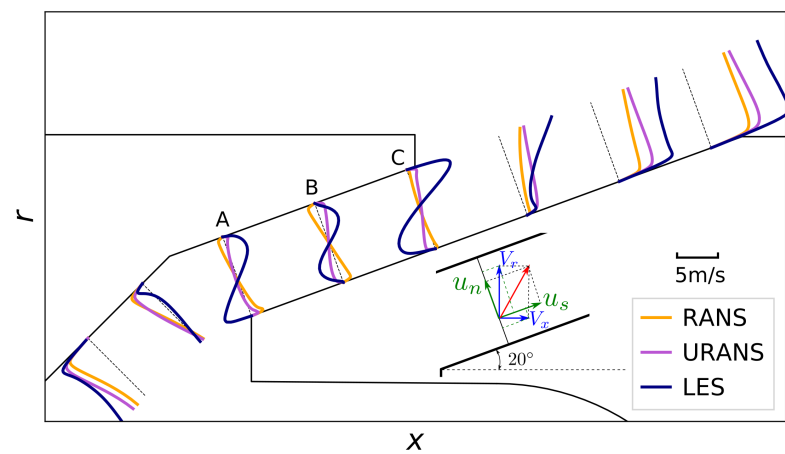

(a)

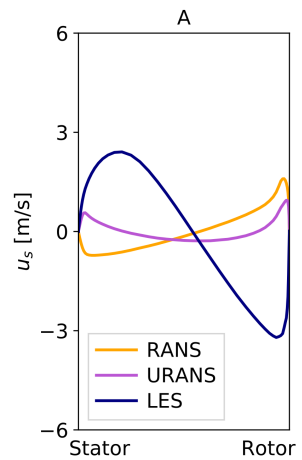

Stator
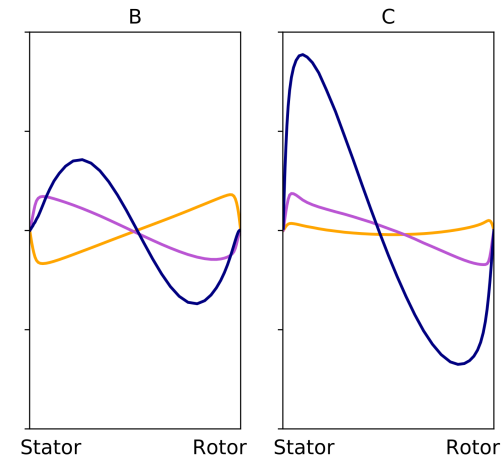

(b)

Figure 8: Mean streamwise velocity profiles $u_{s}$ within the seal gap. (a) view on the geometry; (b) zoom on the three labelled sampling lines.

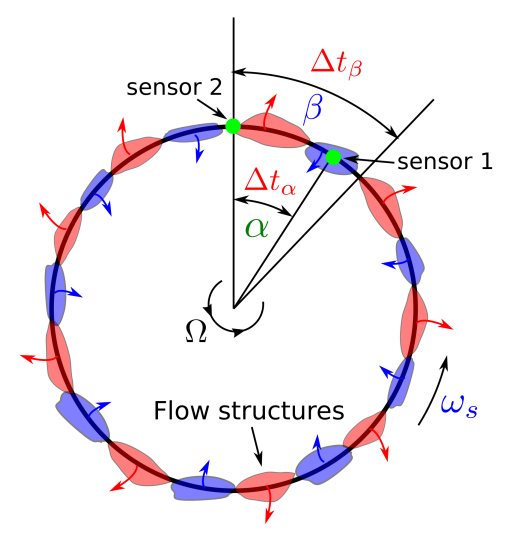

Figure 9: Illustration of cavity flow structures.

\subsubsection{Mean characteristics of unsteady flow structures}

Figure 9 illustrates how a periodic flow structure rotating at angular velocity $\omega_{s}$ will affect a pressure measurement at two stationary sensors at the same radius but at circumferential locations differing by a known angle $\alpha$. If the flow structure has $N$ periodic pairs of perturbation cells, the angle between each flow structure is $\beta=2 \pi / N$ radians. The sensors 1 and 2 will both record the same frequency $(f)$ with a phase lag $\Delta t_{\alpha}$, corresponding to the time it takes for a flow structure to travel between the two sensors. The angular frequency (peak frequency by PSD) detected by each sensor is $f=2 \pi / \Delta t_{\beta}$, where $\Delta t_{\beta}=\beta / \omega_{s}$ is the time taken for a flow feature to pass through an angle $\beta$. These relations give four equations with the six unknown parameters: $f, N, \omega_{s}, \beta, \Delta t_{\alpha}$ and $\Delta t_{\beta}$. The measured frequency $f$ and the phase between the pressure signals detected at the two sensors are required to determine the number of lobes $N$ and angular velocity $\omega_{s}$ of the unsteady flow structure. Generally, the angle $\alpha$ between the two sensors is not always smaller than the angle $\beta$ between two pairs of perturbation cells (as shown in Fig. 9). The determination of the phase difference is further complicated since cross correlation of the two pressure signals will show multiple peaks for the phase lag $\Delta t_{\alpha}$ corresponding to the $N$ lobes, such that $\Delta t_{\alpha}=\alpha / \omega_{s}+n \Delta t_{\beta}$ where $n$ is any integer.

Prior to cross correlations of signals, the measured unsteady pressure data were further filtered to remove components outside the area of interest around $f=23.4 \Omega$ by a $3^{\text {rd }}$-order Butterworth bandpass filter. The same filter was applied to the LES results focusing on frequencies around $f=23.5 \Omega$ or $f=36.5 \Omega$. Cross correlation was performed on each possible pair of sensors for the experimental results, while it was only applied to two sensors with a circumferential spacing of $5^{\circ}$ for the LES results. An example, illustrated in 
Fig. 10 (a), shows the cross correlation of the pressure data between the sensors 1006 and $1002\left(10^{\circ}\right.$ spacing $)$ over one rotor rotation. A python pseudo-code for the cross correlation is attached in Appendix A. The phase lag (time lag) between the two signals is non-dimensionalised by one rotor revolution time. Evenly distributed peaks of the cross correlation coefficient are clearly seen in the figure, indicating a periodic rotating flow structure. If, for a flow structure spinning at the same direction as the rotor, the angle between perturbation cells $(\beta)$ is greater than the angle between two pressure sensors $\left(\alpha=10^{\circ}\right)$ then the time difference from the origin (0) to the first positive peak with positive time lag is expected to correspond to the time taken for a perturbation cell to travel through angle $\alpha$ (between the two probes). The interval between two adjacent positive peaks corresponds to the time lag between two neighbouring flow structures.

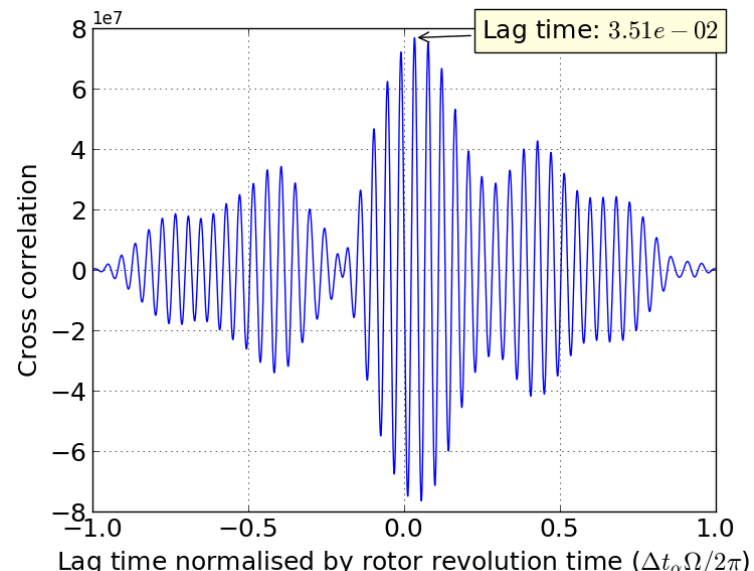

(a)

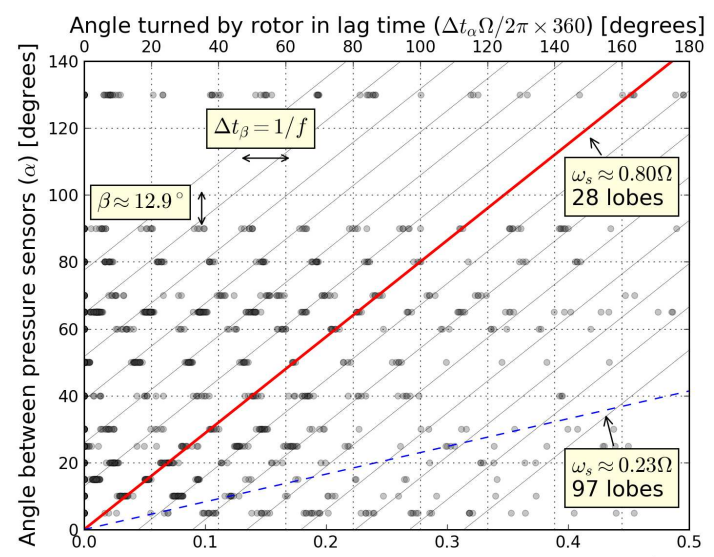

(b)

Figure 10: (a) cross correlation of pressure between sensors 1006 and 1002 over one rotor revolution, (b) summary plot of the cross correlation results.

Cross correlations of pressure data, for each combination of the 6 sensors (15 pairs in total), have been repeated for each of the 116 rotor revolutions. A summary plot of all the cross correlation results is shown in Fig. 10 (b). The angle between the sensor pairs ( $\alpha$, shown on the vertical axis) is plotted against the peak time lag $\left(\Delta t_{\alpha} \Omega / 2 \pi\right)$ collected from each rotor revolution. The peak time lag can also be denoted as the angle turned by the rotor in $\Delta t_{\alpha}$, shown on upper horizontal axis. On the map, symbols are plotted in partial transparency. That means that the more samples drop in the same region, the darker they will appear, and vice versa. Beginning from the origin, a straight line must link as many symbols as possible to signify a periodically rotating flow structure. If, for a lobed flow structure spinning at the same direction as the rotor, the angle between flow features $(\beta)$ is greater than the angle between two sensors $\left(e . g ., \alpha=5^{\circ}\right)$ then a straight line should traverse the origin and the first group of symbols at $\alpha=5^{\circ}$ and pass through symbol groups at other sensor spacing angles as well. This gives the straight solid line in red in Fig. 10 (b). Its slope indicates the speed of the flow structure $\left(\omega_{s} \approx 0.80 \Omega\right)$, and the flow structure has 29 lobes. It is also observed that nearly all of the symbols lie on straight lines parallel to the red one with a constant interval, which shows the flow structure at different phases. The vertical interval corresponds to the angle between two flow features $\left(\beta=12.3^{\circ}\right)$, and the horizontal interval corresponds to the lag time between two adjacent flow structures $\left(\Delta t_{\beta}=1 / f\right)$. An alternative to the red solid line is given by the blue dashed line, then the angle interval between two perturbation cells is smaller than the angle between the sensors at $5^{\circ}$ spacing. Hence, the straight line must connect the origin and the second positive symbol group at $\alpha=5^{\circ}$ and pass as many samples at other sensor spacings as possible. This gives a flow structure with 102 lobes rotating at $23 \%$ of the rotor speed. As fewer symbols are found on the blue dashed line, this possibility is less likely to the one with 29 lobes and rotating at $80 \%$ disc speed. The characteristic parameters of the flow structure are also listed in Tab. 3 for further comparison.

In the LES results, two distinct peaks are found at $f / \Omega=23.5$ and 36.5. The same filtering and cross-correlation procedures are applied respectively to these two peak frequencies to obtain their rotating 
speed and number of flow structures. As noise is less pronounced in the LES than in the experiment, cross correlations are applied to each of the 8 rotor revolutions. The averaged cross correlation reveals $\Delta t_{\alpha} \Omega / 2 \pi=3.19 \times 10^{-2}$ for $f / \Omega=23.5$, and $\Delta t_{\alpha} \Omega / 2 \pi=3.09 \times 10^{-2}$ for $f / \Omega=36.5$. Using the formula in Eqs. (3)-(5), one can compute the speed of the flow structure and the number of lobes. The results are listed in Tab. 3. 54 and 81 lobes, spinning at $43.5 \%$ and $44.9 \%$ of the disc speed, are identified for the two peak frequencies $f / \Omega=23.5$ and 36.5, respectively. These correspond to 2 and 3 lobed flow structures within the $13.33^{\circ}$ computational sector. The results reveal that there are sometimes 54 and sometimes 81 lobed perturbation cells rotating at a speed close to $44 \% \Omega$ for the entire annulus. This will be discussed further in the next section.

$$
\begin{gathered}
\omega_{s}=\alpha / \Delta t_{\alpha} \\
\beta=\omega_{s} \Delta t_{\beta}=\omega_{s} / f \\
N=2 \pi / \beta
\end{gathered}
$$

Table 3: Parameters of the flow structures.

\begin{tabular}{lllll}
\multicolumn{6}{c}{ Table 3: Parameters of the flow structures. } \\
\hline Case & Peak frequency $(f / \Omega)$ & Speed $\left(\omega_{s}\right)$ & N. of lobes $(N)$ & Extent of lobe $(\beta)$ \\
\hline \multirow{2}{*}{ Experiment } & 23.4 & $80.0 \% \Omega$ & 29 & $12.41^{\circ}$ \\
\hline \multirow{2}{*}{ URANS } & 23.5 & $43.5 \% \Omega$ & 54 & $6.67^{\circ}$ \\
& 36.5 & $44.9 \% \Omega$ & 81 & $4.44^{\circ}$ \\
& 16.2 & $45.0 \% \Omega$ & 36 & $10.0^{\circ}$ \\
\hline
\end{tabular}

The URANS solution has been analysed following the same procedures. Verification of the PSD and cross correlation is made with flow visualisation as URANS results show very clear periodicities. The parameters of the URANS flow structure are given in Tab. 3. The flow structures associated with the two peak frequencies rotate at the speeds $\omega_{s}=45.0 \% \Omega$ and $\omega_{s}=45.2 \% \Omega$, respectively. Finally, 48 and 36 lobes have been identified for the two peak frequencies. Another two URANS simulations were conducted with larger inner time step of dual-time stepping scheme but are not presented here. However some sensitivity of the URANS results should be noted. With larger inner time steps which give a bigger speed-up ratio, the sub convergence level reduces, and the frequency of the unsteady flow changes as well. Furthermore, unsteadiness was found to initialise later for the case with lower sub convergence level. This indicates that the URANS prediction of rim seal flows depends on the sub convergence.

\subsubsection{Instantaneous features of unsteady flow structures}

In order to investigate the instantaneous unsteady flow features and further confirm the lobe number and rotational speed obtained from PSD and cross correlation, the LES instantaneous flow fields are presented and discussed.

The instantaneous flow fields are extracted on the three sampling lines (A, B and C) described in Fig. 7 (a) for two time instants, as shown in Fig. 11. On each extracted slice, the bottom edge is on the rotor surface, while the top edge is on the stator wall. The horizontal axis is the azimuthal angle $\theta$, and $\theta$ increases from right to left following the rotor's rotational direction. The contour colour indicates the magnitude of the streamwise velocity. The arrows represent the 2-D fluctuating velocity vectors relative to their ensemble averaged values within the extracted plane.

Ingestion and egestion are clearly observed through the streamwise velocity contour map, i.e. negative $u_{s}$ associates with ingestion and positive $u_{s}$ indicates egestion. In Fig. 11 (a), two lobes are found in the contour map. In Fig. 11 (b), three lobes are observed within the simulated sector. These confirm the unsteady flow structures derived from PSD and cross correlation in the previous section. Another observation made on the same map is that when egestion occurs, the fluctuating velocity vectors follow the rotor's rotating direction, 


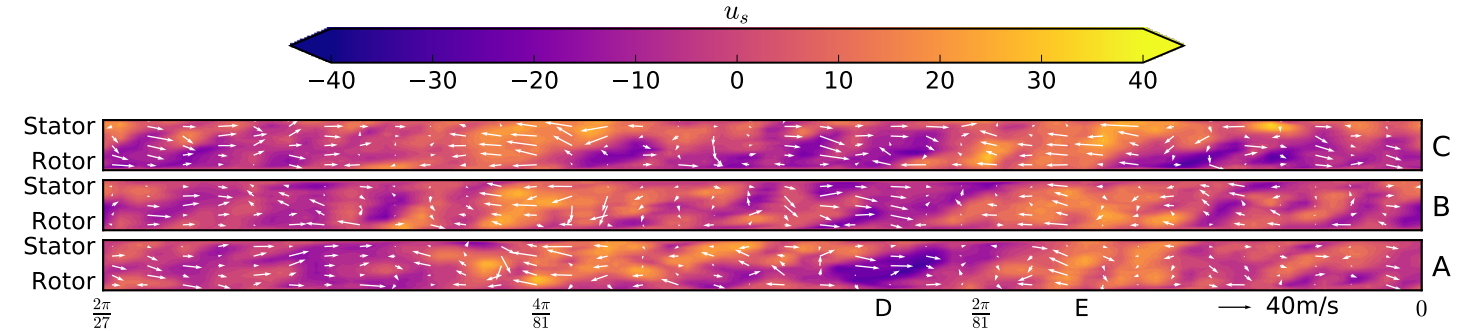

(a)

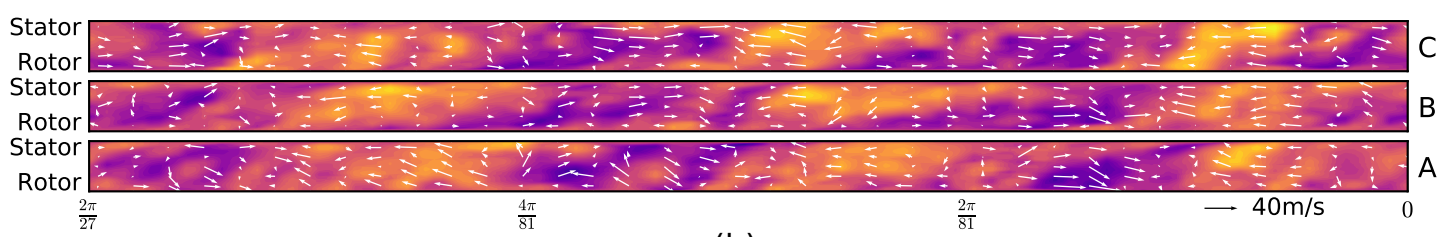

(b)

Figure 11: Streamwise velocity contours, as well as the fluctuating velocity, on the three measurement stations described in Fig. 7 (a). (a) 2 lobes; (b) 3 lobes.

and vice versa. This means that the ingestion and egestion unsteady flow structures could also be observed through tangential velocity. Ingestion associates with a high swirl region, while egestion corresponds to a low swirl velocity zone. In addition, the velocity fluctuation amplitude, as indicated by the contour colour and vector scale, is considerably greater than the mean streamwise velocities shown in Fig. 8. This illustrate the importance of the unsteady flow features.

It seems, as observed in Fig. 11, that flow undergoes an azimuthal phase shift moving from plane A to plane C. The flow on plane C leads that on plane A. Indeed, this is confirmed by further analysis. As the lobes of the flow structure are observed throughout the area between the rotor and stator surfaces, results along the line mid-way between the stator and rotor surfaces $\left(x=0.5\left(x_{\text {stator }}+x_{\text {rotor }}\right)\right)$ from Fig. 11 are considered representative, and are plotted in Fig. 12. The fluctuating streamwise velocities from the three locations, are staggered at 0,40 and $80 \mathrm{~m} / \mathrm{s}$ along the vertical axis for clarity. The phase lags between the lines $\mathrm{A}$ and $\mathrm{C}$ are computed using cross correlation. They are about $0.18^{\circ}$ and $0.09^{\circ}$ for the two-lobe and the three-lobe instants, respectively. The phase lags are plotted in the figure through skew lines. Their relation to the qualitative nature of the flow structures is considered below.

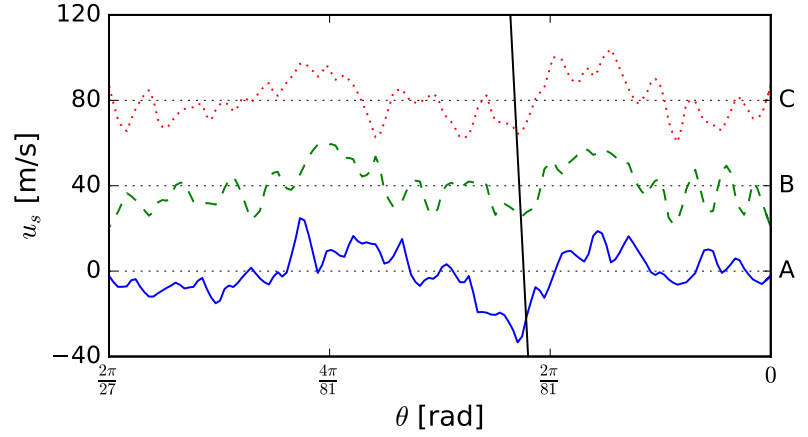

(a)

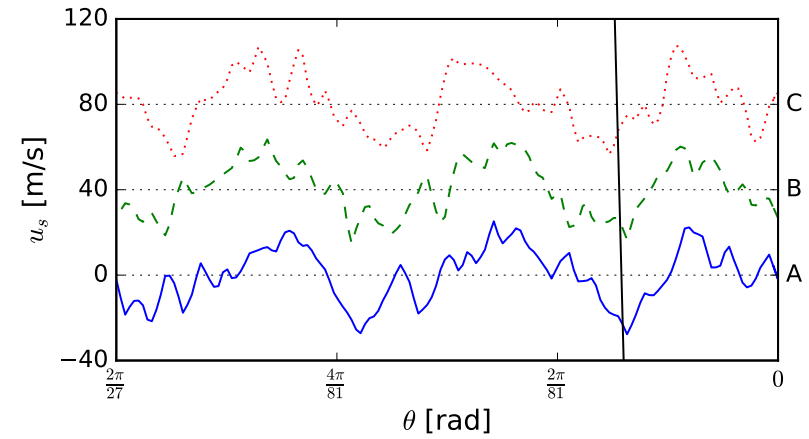

(b)

Figure 12: Streamwise velocity in the middle of the rotor and stator surfaces $\left(x=0.5\left(x_{\text {stator }}+x_{\text {rotor }}\right)\right)$, from the three slices plotted in Fig. 11. (a) 2 lobes; (b) 3 lobes.

Considering a sketch of the unsteady flow structure proposed in Fig. 13, the upper part of the flow 


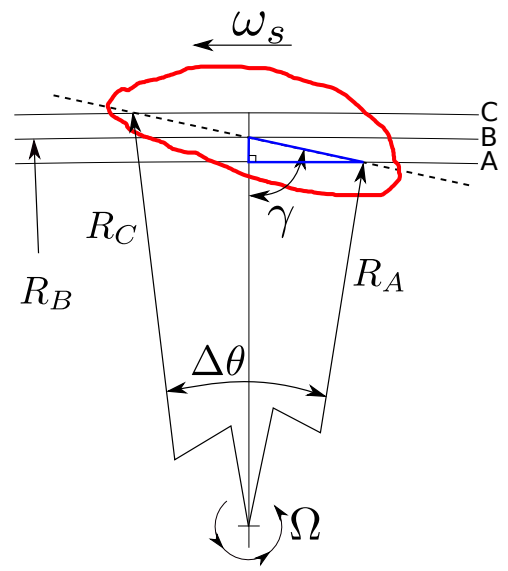

Figure 13: Schematic of the unsteady flow structures' shape in on a plane perpendicular to the shaft axis.

structure is ahead of its lower part as observed in Fig. 12. As the radial distance between the extracting locations ( $\mathrm{A}, \mathrm{B}$ and $\mathrm{C}$ ) is very small compared with their radii and the phase lag between $\mathrm{A}$ and $\mathrm{C}$ is also small (less than $0.2^{\circ}$ ), the extracting lines A, B and $\mathrm{C}$ can be considered as parallel straight lines. Thus, an angle $(\gamma)$ of the unsteady flow structure to the radial direction can be derived with the help of the highlighted right-angled triangle. Applying Fig. 6 to the two instantaneous flow fields in Fig. 12, the angle $\gamma$ is obtained for both the two instants: $\gamma \approx 40.5^{\circ}$ for the case with 2 lobes within the simulated sector, and $\gamma \approx 23.2^{\circ}$ for the three-lobed structure. The value of $\gamma$ implies that the orientation of the flow structure is between the radial and azimuthal directions, and turns towards the radial direction if more lobes are involved within the annulus.

$$
\gamma=\arctan \left(\frac{0.5 \Delta \theta R_{C}}{R_{B}-R_{A}}\right)
$$

It is also of interest to investigate the flow behaviour on meridian planes. Three characteristic circumferential positions are chosen in Fig. 11 (a): D for ingestion, E for egestion and $\frac{2 \pi}{81}$ on the edge of ingestion and egestion. The circumferential vorticity component and 2-D velocity vectors on the three planes are plotted in Fig. 14. In Fig. 14 (a), ingestion takes place. The fluid from the main annulus separates into two streams, one stream goes into the cavity through the rim seal clearance forming ingestion, the other stream flows downstream in the main gas-path. Within the rim seal gap, a positive vorticity contour is observed near the rotor surface, while a negative vorticity contour is seen near the stator. Egestion can be easily identified through the 2-D velocity vectors in Fig. 14 (b). In contrast to ingestion, positive vorticity is detected near the stator and negative value appears near the rotor. On the edge of ingestion and egestion, as shown in Fig. 14 (c), positive and negative vorticity zones are observed near both the stator and rotor side. This shows further similarity with Taylor-Couette type flow.

Finally, the ingestion (position D) and egestion (position E) velocity profiles are plotted together with the ensemble averaged velocity profiles $\left\langle u_{s}\right\rangle$ in Fig. 15, within the rim seal gap. The unsteady streamwise velocities are large compared with their ensemble averaged values. The ingestion and egestion phenomena might be dominated by the unsteady effects rather than the mean velocity profiles.

\section{Conclusions}

The modified Hydra LES code has been validated against a DNS database for pipe flow, and utilised for simulations of a chute rim seal flow of a sealed rotor/stator cavity, comparing with experimental data, RANS and URANS solutions. LES achieved the best agreement with the measured mean pressure distribution, and URANS appears to slightly improve the steady RANS prediction. Examination of tangential velocity profiles within the overlapping seal gap explains the different prediction of the pressure distribution from 

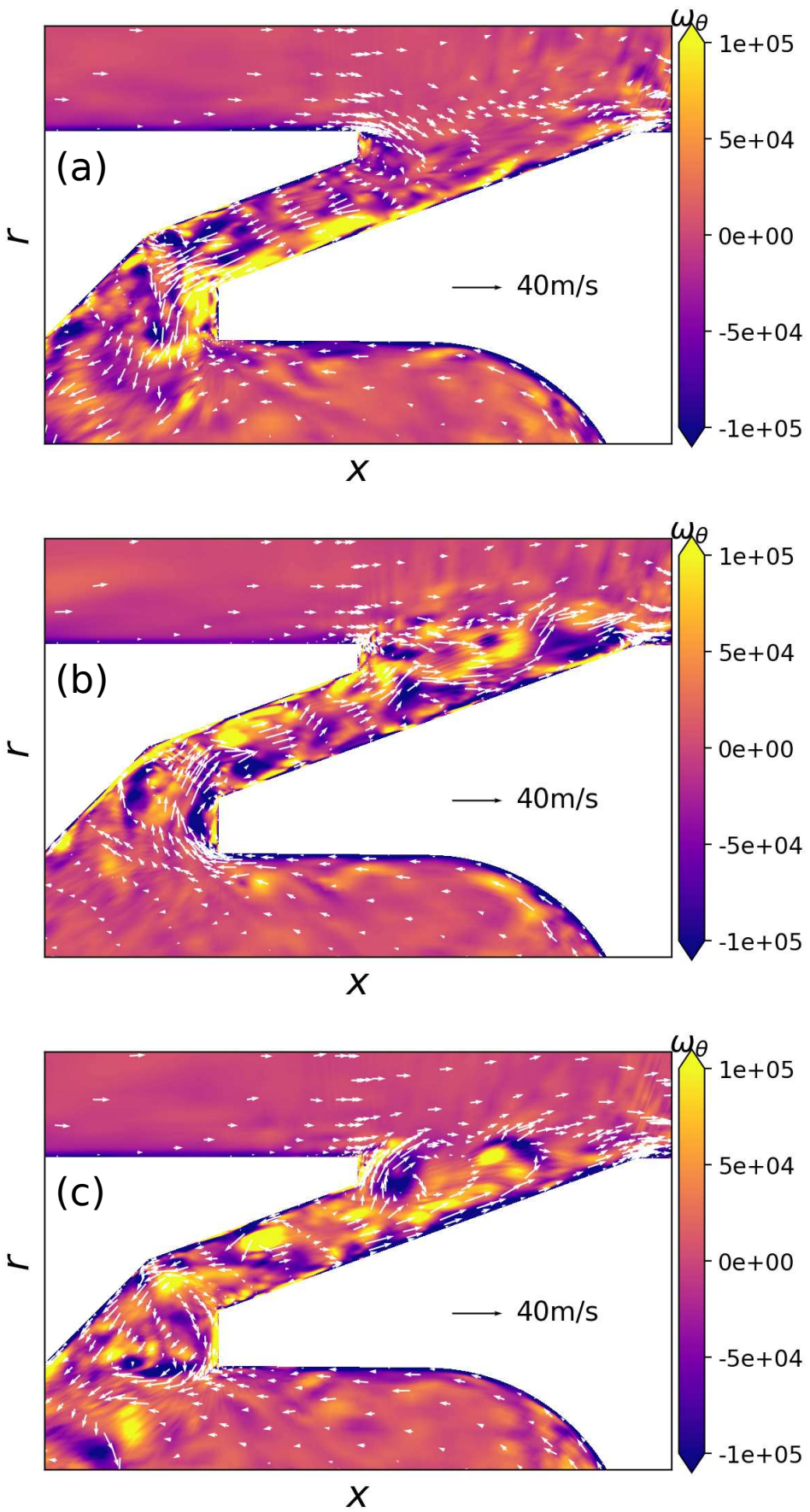

Figure 14: Circumferential vorticity component Contours with 2-D velocity vectors on meridian planes. Extracting positions are described in Fig. 11 (a). (a) Ingestion (D); (b) Egestion (E); (c) Edge ( $\left.\frac{2 \pi}{81}\right)$. 


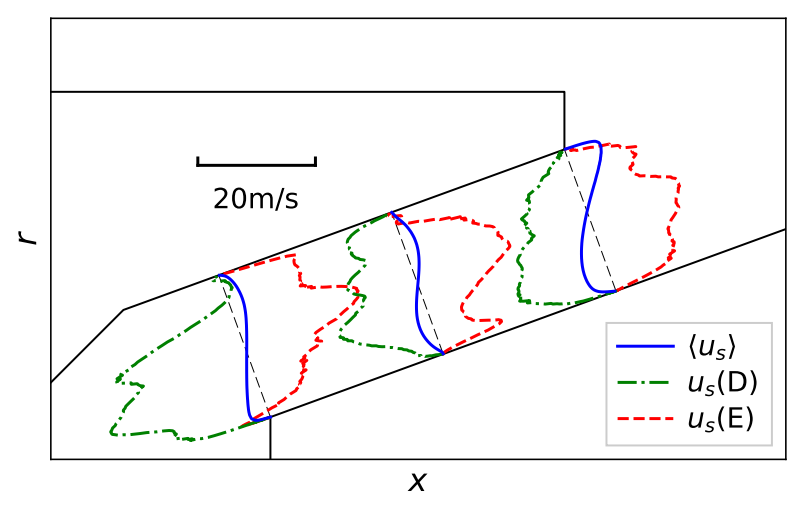

Figure 15: Ensemble averaged and unsteady streamwise velocity profiles $u_{s}$.

the different methods. A mean clockwise vortex has been identified within the seal clearance in the LES which is in contrast to the RANS results and classic rotor-stator cavity flow. This may be interpreted as evidence of Taylor-Couette type flow in the overlapping rim seal clearance.

Large scale unsteady flow structures are observed in the experiment and the CFD. The distinct peak frequency at $f / \Omega=23.4$ observed in the experiment has been accurately captured by LES. This encourages use of LES for rim seal flow investigations. However there are some significant discrepancies between the experiment and LES, with 29 lobed structures rotating at $80 \% \Omega$ in the measurement, and 54 or 81 features spinning at about $44 \% \Omega$ for LES. The differences may be caused by the limited sector angle or other approximations. The analysis of instantaneous flow fields provides further confidence in results deduced from PSD and cross correlation procedures for both CFD and experiment. Further investigation of the instantaneous flow fields illustrates qualitatively the shape of the unsteady flow structures. The outer part of a unsteady flow structure (at higher radius) is ahead of, in azimuthal phase, its inner part (at lower radius). This phase lag helps, qualitatively, describes the orientation of unsteady flow structure which is oriented between the radial and azimuthal directions, and moves towards radial alignment if more lobes are involved within the annulus. Evidence of Taylor-Couette type flow appears again through an instantaneous flow field on an azimuthal plane between ingestion and egestion zones.

URANS using a dual-time stepping scheme with a $30^{\circ}$ sector captures some unsteadiness but the peak frequency did not match the experimental one. This approach shows dependency of the solutions on the sub convergence level.

\section{Nomenclature}

\section{Roman Symbols}

$b \quad$ Rotor disc rim radius

$0.2367[\mathrm{~m}]$

$C_{p} \quad$ Nondimensional pressure

$\frac{\langle p\rangle-\left\langle p_{1}\right\rangle}{0.5 \rho \Omega^{2} b^{2}}[-]$

$C_{s} \quad$ Smagorinsky model coefficient

0.08 [-]

$C_{w} \quad$ Nondimensional flow rate

$f \quad$ Frequency

$L \quad$ Length of pipe

$\dot{m}_{c} \quad$ Coolant mass flow rate 
$N \quad$ Number of unsteady flow structures in the entire annulus

$p \quad$ Static pressure

$R \quad$ Radius of pipe

$4.17 \times 10^{-3}[\mathrm{~m}]$

$r \quad$ Radius

$R e_{b} \quad$ Reynolds number based on pipe diameter and bulk velocity

$R e_{\phi} \quad$ Rotational Reynolds number

$R e_{\tau} \quad$ Reynolds number based on pipe radius and friction velocity

$u_{b} \quad$ Pipe flow bulk velocity

$u_{s}, u_{n}, u_{\theta}$ Streamwise, wall-normal and tangential velocity components in rim seal coordinates

$u_{\tau} \quad$ Friction velocity

$u_{z} \quad$ Pipe flow streamwise velocity

$x \quad$ Axial coordiante

\section{Greek Symbols}

$\alpha \quad$ Angle between two pressure sensors

$\beta \quad$ Angle between two adjacent flow structures

$\Delta(r \theta)^{+}$Nondimensional distance in the azimuthal direction in wall units

$\Delta t_{\alpha} \quad$ Time lag of pressure signals between two azimuthal pressure probes

$\Delta t_{\beta} \quad$ Time lag between two adjacent flow structures

$\Delta x^{+} \quad$ Nondimensional distance in $x$ direction in wall units

$\Delta y^{+} \quad$ Nondimensional wall distance in wall units

$\epsilon \quad$ Weighting coefficient of numericial viscosity in Roe scheme

$\gamma \quad$ Angle of flow structure to radial direction

$\mu \quad$ Molecular viscosity

$1.81 \times 10^{-5}[\mathrm{~kg} /(\mathrm{ms})]$

$\Omega \quad$ Rotor's angular speed

$\frac{2 \pi \times 7000}{60}[\mathrm{rad} / \mathrm{s}]$

$\omega_{s} \quad$ Speed of unsteady flow strucutres

$\omega_{\theta} \quad$ Circumferential vorticity component

$\rho \quad$ Density

$\theta \quad$ Circumferential angle

$\xi \quad$ Random number 


\section{Superscripts}

$+\quad$ Nondimensional quantity

' Fluctuating component

\section{Other symbols}

$\langle\cdot\rangle \quad$ Ensemble average

\section{Acronymus}

CFD Computational fluid dynamics

CFL Courant-Friedrichs-Lewy condition

LES Large-eddy simulation

OPLUS Oxford parallel library for unstructured solver

ORF Oxford rotor facility

PSD Power spectral density

RANS Reynolds-averaged Navier-Stokes

SGS Subgrid-scale

URANS Unsteady RANS

\section{Acknowledgements}

Funding for this research from Rolls-Royce plc and support from colleagues is gratefully acknowledged. In particular, we thank Matthew Miller, Peter Smout and Sebastiaan Bottenheim of Rolls-Royce.

\section{References}

[1] B. V. Johnson, G. J. Mack, R. E. Paolillo, W. A. Daniels, Turbine rim seal gas path flow ingestion mechanisms, AIAA Paper 94.

[2] P. D. Smout, J. W. Chew, P. R. N. Childs, ICAS-GT: A European collaborative research programme on internal cooling air systems for gas turbines, in: Proceedings of the ASME Turbo Expo 2002: Power for Land, Sea, and Air, Amsterdam, The Netherlands, 2002, no. GT2002-30479.

[3] D. E. Bohn, A. Decker, H. Ma, M. Wolff, Influence of sealing air mass flow on the velocity distribution in and inside the rim seal of the upstream cavity of a 1.5-stage turbine, in: Proceedings of the ASME Turbo Expo 2003, collocated with the 2003 International Joint Power Generation Conference, Atlanta, Georgia, USA, 2003, no. GT2003-38459. URL http://dx.doi.org/10.1115/GT2003-38459

[4] C. Cao, J. W. Chew, P. R. Millington, S. I. Hogg, Interaction of rim seal and annulus flows in an axial flow turbine, Journal of Engineering for Gas Turbines and Power 126 (4) (2004) 786-793, (Also conference paper No. GT2003-38368 in Proceedings of the ASME Turbo Expo 2003, collocated with the 2003 International Joint Power Generation Conference).

[5] V. Autef, Computation of disc cavity rim sealing, Tech. Rep. University of Surrey/Institut National Polytechnique de Grenoble project report (2002).

[6] J. W. Chew, N. J. Hills, C. Hornsby, C. Young, Recent developments in application of CFD to turbomachinery internal air systems, in: 5th European Turbomachinery Conference (ETC5), Prague, 2003.

[7] R. Jakoby, T. Zierer, K. Lindblad, J. Larsson, L. de Vito, D. E. Bohn, J. Funcke, A. Decker, Numerical simulation of the unsteady flow field in an axial gas turbine rim seal configuration, in: Proceedings of the ASME Turbo Expo 2004: Power for Land, Sea, and Air, Vienna, Austria, 2004, no. GT2004-53829. doi:10.1115/GT2004-53829. URL http://dx.doi.org/10.1115/GT2004-53829

[8] J. Boudet, V. N. D. Autef, J. W. Chew, N. J. Hills, O. Gentilhomme, Numerical simulation of rim seal flows in axial turbines, Aeronautical Journal 109 (1098) (2005) 373-383. 
[9] T. S. D. O’Mahoney, N. J. Hills, J. W. Chew, T. Scanlon, Large-eddy simulation of rim seal ingestion, Proceedings of the Institution of Mechanical Engineers, Part C: Journal of Mechanical Engineering Science 225 (12) (2011) $2881-2891$. doi:10.1177/0954406211409285.

URL http://journals.sagepub.com/doi/abs/10.1177/0954406211409285

[10] O. J. P. Gentilhomme, Turbine rim seal ingestion, Ph.D., University of Sussex (2004).

[11] P. Schuepbach, R. S. Abhari, M. G. Rose, J. Gier, Influence of rim seal purge flow on the performance of an endwall-profiled axial turbine, Journal of Turbomachinery 133 (2) (2010) 021011-021011.

[12] M. Chilla, H. Hodson, D. Newman, Unsteady interaction between annulus and turbine rim seal flows, Journal of Turbomachinery 135 (5) (2013) 051024-051024.

[13] M. Rabs, F.-K. Benra, H. J. Dohmen, O. Schneider, Investigation of flow instabilities near the rim cavity of a 1.5 stage gas turbine, in: Proceedings of the ASME Turbo Expo 2009: Power for Land, Sea, and Air, Orlando, Florida, USA, 2009, no. GT2009-59965. doi:10.1115/GT2009-59965. URL http://dx.doi.org/10.1115/GT2009-59965

[14] P. F. Beard, F. Gao, K. S. Chana, J. Chew, Unsteady flow phenomena in turbine rim seals, Journal of Engineering for Gas Turbines and Power 139 (3) (2016) 032501-032501.

[15] S. S. Savov, N. R. Atkins, S. Uchida, Comparison of single and double lip rim seal geometry, in: Proceedings of the ASME Turbo Expo 2016: Turbomachinery Technical Conference and Exposition, ASME, Seoul, South Korea, 2016, no. GT2016-56317.

[16] R. Schädler, A. I. Kalfas, R. S. Abhari, G. Schmid, S. Voelker, Modulation and radial migration of turbine hub cavity modes by the rim seal purge flow, in: Proceedings of the ASME Turbo Expo 2016: Turbomachinery Technical Conference and Exposition, ASME, Seoul, South Korea, 2016, no. GT2016-56661.

[17] J. A. Scobie, C. M. Sangan, J. M. Owen, G. D. Lock, Review of ingress in gas turbines, Journal of Engineering for Gas Turbines and Power 138 (12) (2016) 120801-120801-16. doi:10.1115/1.4033938. URL http://dx.doi.org/10.1115/1.4033938

[18] N. J. Hills, J. W. Chew, A. B. Turner, Computational and mathematical modelling of turbine rim seal ingestion, in: Proceedings of the ASME Turbo Expo 2001: Power for Land, Sea, and Air, New Orleans, Louisiana, USA, 2001 , no. 2001-GT-0204. doi:10.1115/2001-GT-0204.

URL http://dx.doi.org/10.1115/2001-GT-0204

[19] N. J. Hills, Achieving high parallel performance for an unstructured unsteady turbomachinery CFD code, Aeronautical Journal 111 (1117) (2007) 185-193.

[20] P. Spalart, S. Allmaras, A one-equation turbulence model for aerodynamic flows, La Recherche Aérospatiale 4 (1994) 5-21.

[21] A. Jameson, Time dependent calculations using multigrid, with applications to unsteady flows past airfoils and wings, in: 10th Computational Fluid Dynamics Conference, AIAA, Honolulu, HI, 1991.

[22] P. Moinier, Algorithm developments for an unstructured viscous flow solver, Ph.D. thesis, University of Oxford (1999).

[23] D. Amirante, N. J. Hills, Large-eddy simulations of wall bounded turbulent flows using unstructured linear reconstruction techniques, Journal of Turbomachinery 137 (5) (2015) 051006-051006.

[24] P. Moin, J. Kim, Numerical investigation of turbulent channel flow, Journal of Fluid Mechanics 118 (1982) $341-377$.

[25] C. Li, A-priori analysis of LES subgrid scale models applied to wall turbulence with pressure gradients, phdthesis, Ecole Centrale de Lille (Nov. 2013).

[26] G. K. El Khoury, P. Schlatter, A. Noorani, P. F. Fischer, G. Brethouwer, A. V. Johansson, Direct numerical simulation of turbulent pipe flow at moderately high Reynolds numbers, Flow, Turbulence and Combustion 91 (3) (2013) $475-495$.

[27] E. Lenormand, P. Sagaut, L. Ta Phuoc, Large eddy simulation of subsonic and supersonic channel flow at moderate Reynolds number, International Journal for Numerical Methods in Fluids 32 (4) (2000) 369-406.

\section{Appendix}

\section{Appendix A. Python pseudo-script for cross correlation}

Assuming $p s 1$ and $p s 2$ containing pressure data recorded from pressure probes at different circumferential positions and the probe 1 being upstream of probe 2, a python pseudo-script to calculate the time lag between the two probes is given below:

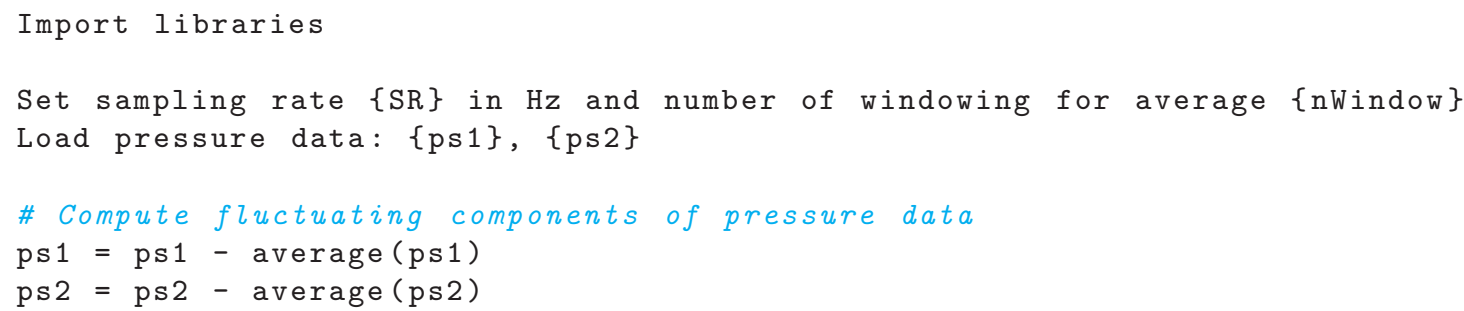




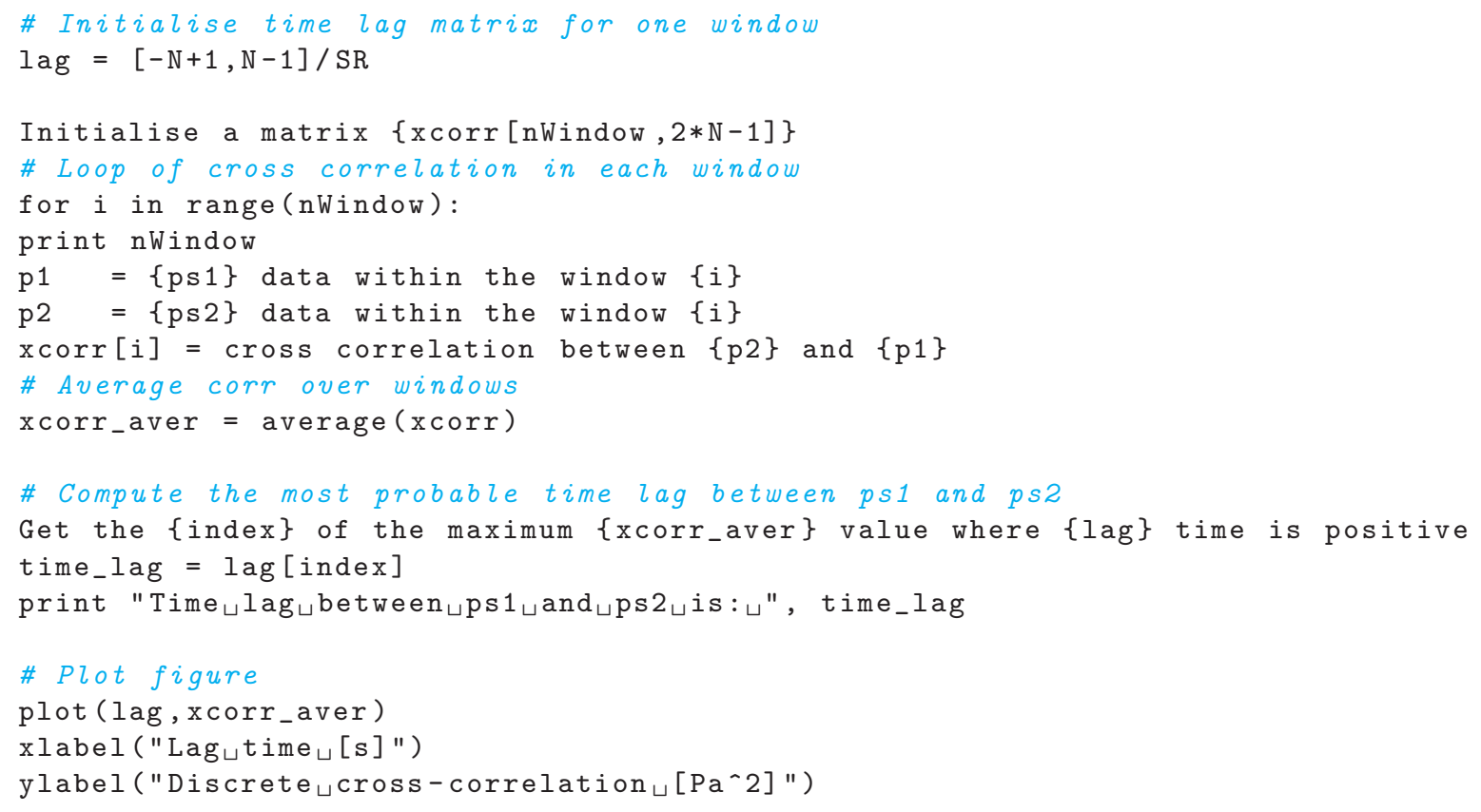

